\title{
SOME TWO-DIMENSIONAL LOCI CONNECTED WITH CROSS RATIOS*
}

\author{
BY
}

\section{J. L. WALSH}

1. Introduction. The writer has recently published a proof of the following theorem: $\dagger$

THEOREM I. If the points $z_{1}, z_{2}, z_{3}$ vary independently and have circular regions as their respective loci, then the locus of the point $z_{4}$ defined by the real constant cross ratio

is also a circular region.

$$
\lambda=\left(z_{1}, z_{2}, z_{3}, z_{4}\right)
$$

It is the purpose of the present paper to consider generalizations of and other results related to Theorem $I$, primarily the determination of the locus of the point $z_{4}$ defined as in Theorem I, when $z_{1}, z_{2}, z_{3}$ vary independently so as to have certain prescribed loci. Thus one may raise the following question: If two variable points lie respectively on two fixed circles, where does the mid-point of their segment lie? The answer is contained in Theorem VI. Or again, if the loci of the points $z_{1}, z_{2}, z_{3}$ are regions each bounded by a number of circles, what can be said of the locus of $z_{4}$ ? The answer is given by Theorem VII.

All the results proved concerning such loci as these can be interpreted in terms of the roots of the jacobian of two binary forms. Such an interpretation is given in $\$ 12$.

The chief method used in the present paper to determine the locus of $z_{4}$ in any given case is that suggested in I (pp. 102, 103, footnote), namely the determination of the locus of $z_{4}$ when $z_{1}$ and $z_{2}$ are held fast and $z_{3}$ varies over its locus; the determination of the locus of this locus of $z_{4}$ when $z_{1}$ is kept fixed but $z_{2}$ varies over its locus; and finally the determination of the locus of this new locus of $z_{4}$ when $z_{1}$ varies over its prescribed locus. In the present paper this method is carried through geometrically. The same method has been used by Professor A. B. Coble $\ddagger$ to determine analytically the locus of $z_{4}$ in Theorem I,

* Presented to the Society, September 7, 1920, and September 9, 1921.

† These T r a n s a c t i o n s, vol. 22 (1921), pp. 101-116; this paper will be referred to as I. We shall also have occasion to refer to another of our papers, using the letter S: Comples Rendus du Congrès International des Mathématiciens, Strasbourg, 1920, pp. 349-352.

The term locus used in Theorem I of the present paper replaces the term envelope used in I.

† Bulletin of the American Mathematical Society, vol.27(1920-1921), pp. 434-437. 
but analytic determination in the more general case is less illuminating and probably more difficult than the geometric determination.

Throughout the present paper, as throughout I, circles play a central rôle.

2. Theorem II, a property of the boundaries of the loci. We suppose once for all that the loci of the points $z_{1}, z_{2}, z_{3}$ are closed regions bounded by a finite number of regular curves.* We admit, however, the possibility of having these loci either points or regular curves instead of two-dimensional continua bounded by such curves.

For loci of this sort we prove a result which corresponds to, but is more general than Lemma III (I, p. 104); the prescribed loci of $z_{1}, z_{2}, z_{3}$ are denoted by $T_{1}, T_{2}$, $T_{3}$, respectively, and the locus of $z_{4}$ defined as in Theorem $I$ is denoted by $T_{4}$.

THEOREM II. If the point $z_{4}$ is on the boundary of $T_{4}$, then any set of points $z_{1}, z_{2}, z_{3}$ corresponding lie on the boundaries of their respective regions $T_{1}, T_{2}, T_{3}$; if none of these four points is at a vertex of its proper region, the circle $C$ through the points $z_{1}, z_{2}, z_{3}, z_{4}$ cuts the boundaries of $T_{1}, T_{2}, T_{3}, T_{4}$ all at angles of the same magritude, and if $C$ is transformed into a straight line, the lines tangent to these boundaries at the points $z_{1}, z_{2}, z_{3}, z_{4}$, respectively, are parallel. If one or more of the points $z_{1}, z_{2}, z_{3}, z_{4}$ is at a vertex of its proper region, $C$ cuts the boundaries of the other regions at angles of the same magnitude, and if $C$ is transformed into a straight line the lines tangent to these boundaries at the corresponding points are parallel.

If we consider the defining relation to be

$$
\left(z_{1}, z_{2}, z_{3}, z_{4}\right)=\lambda \text {, }
$$

if $\lambda=0,1$, or $\infty$, and if $T_{4}$ coincides with one of the original regions (see I, p. 103), only one of the points $z_{1}, z_{2}, z_{3}$ is effectively concerned with the location of $z_{4}$, and Theorem II is not true. Whenever $T_{4}$ is the entire plane, the locus of $z_{4}$ has no boundary and the theorem has no meaning. These possibilities are henceforth excluded.

We phrase the proof to deal with the case that each of the regions $T_{1}, T_{2}, T_{3}$ is a two-dimensional continuum, buta change in wording rather than of reasoning is all that is required if one or more of these regions is a point. We discuss later the possibility of curves instead of two-dimensional continua.

When any two of the points $z_{1}, z_{2}, z_{3}, z_{4}$ are kept fixed, the relation defining $z_{4}$ becomes a linear relation between the other two points. Thus, when $z_{2}$ and $z_{3}$ are kept fixed, motion of $z_{1}$ over a two-dimensional continuum moves $z_{4}$ over a two-dimensional continuum, so if $z_{4}$ is on the boundary of its locus, $z_{1}$ is also on the boundary of its locus, and similarly for $z_{2}$ and $z_{3}$. Under such a linear correspondence, moreover, a circle corresponds to a circle. For example, when $z_{3}$

\footnotetext{
- For the definition of a regular curve, see Osgood, Funktionentheorie (second edition), pp. $51,324.150$.
} 
and $z_{4}$ are considered fixed and when $z_{1}$ moves along $C, z_{2}$ also moves along $C$. When $z_{1}$ moves from $C$ along the boundary of $T_{1}, z_{2}$ moves from $C$ and makes the same angle with $C$ as the angle between $C$ and the boundary of $T_{1}$. If the boundary of $T_{2}$ does not make this same angle with $C$, and if neither $z_{1}$ nor $z_{2}$ is at a vertex of the corresponding boundary, this motion of $z_{1}$ in one sense or the other from $C$ will cause $z_{2}$ to move into the interior of $T_{2}$. Then we keep $z_{1}$ and $z_{3}$ fixed. Motion of $z_{3}$ over a two-dimensional continuum all of which is interior to $T_{2}$ will cause $z_{4}$ to move over a two-dimensional continuum all of which is interior to $T_{4}$, so $z_{4}$ cannot be on the boundary of $T_{4}$.

Thus the boundaries of $T_{1}, T_{2}, T_{3}$ all make the same angle with $C$, and if we keep $z_{1}$ and $z_{2}$ fixed, moving $z_{3}$ and $z_{4}$, we see by consideration of the angles at these latter two points that the boundaries of $T_{3}$ and $T_{4}$ make the same angle with $C$. The direct conformality of the angles in every case here shows that when $C$ is transformed into a straight line the tangents to the boundaries of $T_{1}, T_{2}$, $T_{3}, T_{4}$ at $z_{1}, z_{2}, z_{3}, z_{4}$ are parallel if these points are not at vertices of those boundaries. If one or more of the points $z_{1}, z_{2}, z_{3}, z_{4}$ is at a vertex of the corresponding boundary, the result always holds for such of those points as are not at vertices of those boundaries.

If one of the original regions $T_{1}, T_{2}, T_{3}$ is a curve instead of a point or a twodimensional continuum, further proof is necessary. If for example $T_{1}$ and $T_{2}$ are curves and if $C$ does not cut them at $z_{1}$ and $z_{2}$ at equal angles, let us transform $z_{3}$ to infinity. Denote by $C_{2}^{\prime}$ the curve of all points $z_{4}$ which correspond to a particular point $z_{1}$ of $T_{1}$ and to $z_{3}$, as $z_{2}$ traces $T_{2}$. The angle between $C$ and $C^{\prime}{ }_{2}$ at $z_{4}$ is the same as the angle between $C$ and $C_{2}$ at $z_{2}$. When $z_{1}$ now moves on $T_{1}$, points of $C^{\prime}{ }_{2}$ which correspond to particular points $z_{2}$ of $T_{2}$ move in the direction of motion of $z_{1}$. If $C$ does not cut $T_{1}$ and $T_{2}$ at the same angle, this motion of $z_{1}$ on $T_{1}$ will therefore cause $C_{2}^{\prime}$ to sweep out an entire two-dimensional continuum in the neighborhood of $z_{4}$ and hence $z_{4}$ cannot be on the boundary of $T_{4}$.

To compare the angles in which $C$ cuts $T_{1}$ and the boundary of $T_{4}$, we hold $z_{2}$ and $z_{3}$ fast. Motion of $z_{1}$ from $C$ along $T_{1}$ causes $z_{4}$ to move from $C$ along a curve through $z_{4}$ either tangent to $C$ or extending on both sides of $C$. But all points of this curve are points of $T_{4}$, and the curve cuts $C$ at the same angle as $T_{1}$. Hence either $T_{4}$ has a vertex at $z_{4}$ or its boundary cuts $C$ at the same angle as does $T_{1}$. We have now given all the essential reasoning in the proof of Theorem II.

We remark incidentally that curves related as are the curves bounding these regions $T_{1}, T_{2}, T_{3}, T_{4}$ have interesting geometric properties. ${ }^{*}$ Thus when the

- In three-dimensional space we may consider the analogous problem of finding the locus of points $z_{4}$ determined by their real constant cross ratio with the points $z_{1}, z_{2}$, $z_{3}$ whose loci are respectively either three surfaces $S_{1}, S_{2}, S_{2}$ or three regions bounded by these three surfaces; the term cross ratio is to be interpreted precisely as in the plane-the four points $z_{1}, z_{2}, z_{3}, z_{4}$ are to be concyclic and the cross ratio can be defined by any complex coördinate system in 
region $T_{3}$ is simply the point at infinity, the bounding curves $C_{1}, C_{2}, C_{4}$ are so related that $C$ (in this case a line) sets up a correspondence between those curves, so that tangents to $C_{1}$ and $C_{2}$ at corresponding points are parallel and the corresponding points $z_{1}, z_{2}, z_{4}$ on the line $C$ divide $C$ in a constant ratio. Whenever three curves $C_{1}, C_{2}, C_{4}$ have this property, it is true that lines tangent to these curves at corresponding points $z_{1}, z_{2}, z_{4}$ are parallel, the centers of curvature of $C_{1}, C_{2}, C_{4}$ at $z_{1}, z_{2}, z_{4}$ are collinear and the ratios of the segments cut on the line joining them equal the ratios of the corresponding segments of the line $C$. If the curves $C_{1}$ and $C_{2}$ are parallel, $C_{4}$ is parallel to them.

3. Theorems III and IV; cases where the loci are curves. There are two particularly interesting types of curves $T_{1}, T_{2}, T_{3}$ which may be chosen so that $T_{4}$ shall also be a curve.

THEOREM III. Let $C_{1}$ and $C_{2}$ be two curves which are the loci of the points $z_{1}$ and $z_{2}$ respectively; denote by $C$ the locus of the point

$$
z=\frac{m_{2} z_{1}+m_{1} z_{2}}{m_{1}+m_{2}} \quad, \quad m_{1} m_{2} \neq 0,
$$

which divides the segment $\left(z_{1}, z_{2}\right)$ in the real constant ratio $m_{1}: m_{2}$. A necessary and suttient condition that $C$ be a curve is that $C_{1}$ and $C_{2}$ be segments of two parallel lines.

The sufficiency of the condition is immediate; all points $z$ lie on a certain line parallel to $C_{1}$ and $C_{2}$. The locus $C$ is connected since the loci $C_{1}$ and $C_{2}$ are connected (see I, p. 103). Hence $C$ is a curve, a line segment.

To prove the necessity of the condition we resort to the type of reasoning just used in the proof of Theorem II. Let $P$ be an arbitrary point of $C_{1}$, and $C^{\prime}{ }_{2}$ the curve obtained from $C_{2}$ by similarity transformation with center $P$. When $P$ varies on $C_{1}$ and takes a position $P^{\prime}$, every point $Q$ of $C^{\prime}{ }_{2}$ varies and takes a position $Q^{\prime}$ such that $Q Q^{\prime}$ is parallel to $P P^{\prime}$. According to our hypothesis on $C$, of which $C_{2}^{\prime}$ is a part, and when we allow $P P^{\prime}$ to become smaller and smaller, we find that the tangent to $C^{\prime}{ }_{2}$ at $Q$ is parallel to the tangent to $C_{1}$ at $P$. But $Q$ is an arbitrary point of $C^{\prime}{ }_{2}$; then $C^{\prime}{ }_{2}$ (and consequently $C_{2}$ ) is a curve whose tangent has but a single direction and hence is a straight line segment. On the other

the plane of their circle. If under these conditions the locus of $z_{4}$ is not the whole of space, which is always the case if the arbitrary surfaces $S_{1}, S_{2}, S_{3}$ are sufficiently small and sufficiently remote from each other, simple consideration of the boundary of the locus of $z_{4}$ as in Theorem II gives us the following theorem of pure geometry whtch seems to be new:

The three surfaces $S_{1}, S_{2}, S_{3}$ define a congruence of circles $C$ every circle of which cuts all those surfaces at points $z_{1}, z_{2}, z_{3}$ at the same angle, and such that if $C$ is inverted into a straight line the planes tangent to $S_{1}, S_{2}, S_{3}$ at $z_{1}, z_{2}, z_{3}$ are all parallel. That is, any sphere through $C$ cuts $S_{1}, S_{2}$, $S_{3}$ at $z_{1}, z_{2}, z_{3}$ at equal angles. If any surface $S_{4}$ is defined as the locus of points $z_{4}$ defined by a real constant cross ratio with $z_{1}, z_{2}, z_{3}$, the isogonal property holds for all the surfaces $S_{1}, S_{2}, S_{3}, S_{4}$.

The congruence $C$ is a generalization of the well known normal congruence, for which the cross ratio property is also well known. 
hand, $C_{1}$ is a curve whose tangent at an arbitrary point $P$ is parallet to the line $C_{2}$. Hence $C_{1}$ and $C_{2}$ are segments of parallel lines.

Theorem III is not true for the degenerate values $m_{3}=0$ or $m_{2}=0$. The following theorem is likewise false for the degenerate values of the cross ratio:

THEOREM IV. Let three curves $C_{1}, C_{2}, C_{3}$ be the loci of points $z_{1}, z_{2}, z_{3}$, respectively. Denote by $C_{4}$ the locus of the point $z_{4}$ defined by the real constant cross ratio

$$
\lambda=\left(z_{1}, z_{2}, z_{3}, z_{4}\right) \quad \lambda \neq 0,1, \text { or } \infty .
$$

A necessary and sufficient condition that $C_{4}$ be a curve is that $C_{1}, C_{2}, C_{3}$ be arcs of a single circle having no point common to all.

The sufficiency of the condition is immediate. If two of the points $z_{1}, z_{2}, z_{3}$ coincide, $z_{4}$ coincides with them and hence is on the circle of which $C_{1}, C_{2}, C_{3}$ are arcs. If no two of the original points coincide, $z_{4}$ is concyclic with those three points and hence also on that circle. The locus $C_{4}$ is connected (proved as in I, p. 103) and hence is an arc of that circle.

To demonstrate the necessity of the condition, we notice that $C_{1}, C_{2}, C_{3}$ must be circular arcs; otherwise we could choose suitably the point at infinity on $C_{1}$, for example, and $C_{2}$ and $C_{3}$ would become two curves not parallel lines, which would be in contradiction to Theorem III. If $C_{1}, C_{2}, C_{3}$ are not arcs of a single circle there is a point $P$ which belongs to $C_{1}$, for example, but which does not belong to both of the circles of which $C_{2}$ and $C_{3}$ are arcs. When $P$ is transformed to infinity, $C_{2}$ and $C_{3}$ are not both straight lines, so we have again a contradiction of Theorem III. If the arcs $C_{1}, C_{2}, C_{3}$ of a single circle have a point common to, all the locus of $z_{4}$ is the entire plane instead of a curve. The proof of Theorem IV is thus complete.

4. Theorem $V$, the interest of regions bounded by circular arcs. Theorems I-IV all indicate the central position in this study occupied by circles and circular arcs; this is heightened by the invariance of circles and circular arcs under linear transformation. The following theorem results from Theorem II:

THEOREM V. Let the regions $T_{1}, T_{2}, T_{3}$, each bounded by a finite number of circular arcs, be the loci of points $z_{1}, z_{2}, z_{3}$, respectively. Then the region $T_{4}$ which is the locus of the point $z_{4}$ defined by the real constant cross ratio:

$$
\lambda=\left(z_{1}, z_{2}, z_{3}, z_{4}\right)
$$

is also bounded by a finite number of circular arcs.

Every point of the boundary of $T_{4}$ corresponds to points $z_{1}, z_{2}, z_{3}$ on the boundaries of $T_{1}, T_{2}, T_{3}$ and such that the circle $C$ through the four points cuts the boundaries of $T_{1}, T_{2}, T_{3}$ at vertices or such that the angles between $C$ and the boundaries of $T_{1}, T_{2}, T_{3}$ are equal in magnitude. Corresponding to each set of circles, one a boundary (or in part forming part of the boundary) of each of the 
original regions, there are four circles of the type of $C_{4}$ (Lemma IV, I, p. 105) according as $C$ cuts all three circles at the same angle or a definite one at an angle supplementary to the angle cut on the other two. ${ }^{*}$ Corresponding to each vertex of one of the original regions and two circles, one a boundary of each of the other of the original regions, there are two circles of the type of $C_{4}$ (Lemma IV, I, p. 105), and corresponding to two vertices of two separate original regions and a circle bounding the other region there is but one circle of this type. Since there are but a finite number of circular arcs and of vertices in the boundaries of each of the regions $T_{1}, T_{2}, T_{3}$, we have in all but a finite number of circles whose points can be boundary points of $T_{4}$; every boundary point of $T_{4}$ lies on at least one of these circles. The theorem follows at once. It is also true that if $T_{1}, T_{2}$, $T_{3}$ are bounded by at most a countable infinity of circular arcs, then $T_{4}$ is bounded by at most a countable infinity of circular arcs.

Regions of the sort considered in Theorem $\mathrm{V}$ are particularly interesting if they are bounded by entire circles instead of arcs of circles. We shall study in some detail the loci connected with such regions. The simplest case is that of circular regions, and has already been treated. The next simplest case is that of annular regions. We define an annular region to consist of the points common to two circular regions whose boundaries either have no point in common, or are tangent, or coincide. $\dagger$ An annular region is thus a closed region; every circular region is an annular region.

5. Theorem VI, the locus resulting from three circles; case of a single null circle. A general result concerning loci generated by regions whose boundaries are entire circles is to be proved later (Theorem VII). We now prove a preliminary result:

THEOREM VI. If the loci of the points $z_{1}, z_{2}, z_{3}$ are the circles $S_{1}, S_{2}, S_{3}$, respectively, then the locus $S_{4}$ of the point $z_{4}$ defined by the real constant cross ratio

$$
\lambda=\left(z_{1}, z_{2}, z_{3}, z_{4}\right)
$$

is an annular region.

For the values $\lambda=0,1$ or $\infty$, we must have at least two of the points $z_{1}, z_{2}, z_{3}, z_{4}$. coincident. Thus if $\lambda=0$ the locus of $z_{4}$ is the entire plane or $S_{3}$ according as $S_{1}$ and $S_{2}$ have or have not a point in common. Similar facts hold for the other degenerate values of $\lambda$; compare $I, p .103$. In the future we assume $\lambda$ to have none of these values; then no two of the points $z_{1}, z_{2}, z_{3}, z_{4}$ coincide unless three of them coincide. Furthermore we place ourselves in what we may call the general

* This is true even if the three circles are coaxial; see the detailed proof of Lemma IV, I, p. $105 \mathrm{ff}$.

† It is possible to define annular regions so as to exclude the possibility of tangency of the bounding circles. But under this possible definition Theorem VI is no longer true; compare the results of $\$ 5$. 
situation, where none of the circles $S_{1}, S_{2}, S_{3}$ is a null circle, where no two of these circles are tangent, and where the three circles are not coaxial. We may immediately treat the case that the three original circles have a common point; the points $z_{1}, z_{2}, z_{3}$ may all be considered to lie at that point, $z_{4}$ may lie anywhere in the plane, so its locus is the entire plane.

The fact that $S_{4}$ is a two-dimensional continuum appears from the results of $\S 3$; the fact that the locus $S_{4}$ is connected appears by the reasoning used in I, p. 103.

Choose two arbitrary but distinct points $P_{1}$ and $P_{2}$ on $S_{1}$ and $S_{2}$, respectively, and let us consider the locus of $z_{4}$ defined by

$$
\left(P_{1}, P_{2}, z_{3}, z_{4}\right)=\lambda
$$

when the locus of $z_{3}$ is $S_{3}$. If we transform $P_{1}$ to infinity, $z_{4}$ is a point which divides the segment $P_{2} z_{3}$ internally or externally in a constant ratio. Hence the locus of $z_{4}$ is a non-null circle $S_{4}^{\prime}$. If $P_{1}$ and $P_{2}$ are chosen coincident, $z_{4}$ must coincide with them and its locus is a null circle. Let now $P_{2}$ trace. the circle $S_{2}$, while $P_{1}$ remains fixed. If we determine the locus of all points of the variable circle $S_{4}^{\prime}$ we shall have precisely the locus of $z_{4}$ defined by

$$
\left(P_{1}, z_{2}, z_{3}, z_{4}\right)=\lambda
$$

when $z_{2}$ and $z_{3}$ have as their loci the circles $S_{2}$ and $S_{3}$.

We shall first assume that $S_{2}$ and $S_{3}$ are not coaxial with the null circle $P_{1}$ and that neither passes through $P_{1}$. The family of circles $\alpha$ through $P_{1}$ and cutting $S_{2}$ and $S_{3}$ at equal angles forms a coaxial family; compare the theorem quoted in $\mathrm{I}, \mathrm{p}$. 106. If $P_{1}$ is the point at infinity, this family passes through the external center of similitude of $S_{2}$ and $S_{3}$ if $S_{2}$ and $S_{3}$; are equal in size and $P_{1}$ is the point at infinity, this family is a family of parallel lines. Consider the circle $\alpha$ through the point $P_{2}$ and on $\alpha$ the point $A$ determined by the cross ratio

$$
\left(P_{1}, P_{2}, z_{3}, A\right)=\lambda \text {, }
$$

where $z_{3}$ is the intersection of $\alpha$ with $S_{3}$ such that the tangent to $S_{3}$ at $z_{3}$ becomes parallel to the tangent to $S_{2}$ at $P_{2}$ when the circle $\alpha$ is transformed into a straight line. The point $z_{8}$ is uniquely determined by these conditions except in the particular case that $\alpha$ is orthogonal to $S_{2}$ and $S_{3}$; in this case we determine $z_{3}$ by continuity.

When the circle $\alpha$ varies, the point $A$ traces a circle $\sigma_{1}$, by Lemma IV (I, p. 105). Moreover, the point $A$ is continually on the circle $S_{4}^{\prime}$ corresponding to $P_{1}$ and $P_{2}$, and when $\alpha$ is transformed into a straight line, the line tangent to $S_{1}^{\prime}$ at $A$ is parallel to the line tangent to $S_{3}$ at $z_{3}$. Hence as $\alpha$ varies, $S_{4}^{\prime}$ remains constantly tangent to $\sigma_{1}$; there is a circle $S_{4}^{\prime}$ tangent to $\sigma_{1}$ at every point of $\sigma_{1}$. 
The family of circles $\beta$ through $P_{1}$ and cutting $S_{2}$ and $S_{3}$ at supplementary angles also forms a coaxial family. When $P_{1}$ is the point at infinity the circles $\beta$ all pass through the internal center of similitude of $S_{2}$ and $S_{3}$. When $\beta$ varies, the procedure just employed for $\alpha$ can be used to show that the circle $S_{4}^{\prime}$ remains constantly tangent to a second circle $\sigma_{2}$ at a point of the circle $\beta$ through $P_{2}$; there is a circle $S_{4}^{\prime}$ tangent to $\sigma_{2}$ at every point of $\sigma_{2}$.

The circles $\sigma_{1}$ and $\sigma_{2}$ are distinct. In fact, let us consider $P_{1}$ still as the point at infinity, and denote by $A_{2}$ and $A_{3}$ the centers of $S_{2}$ and $S_{3}$. Then the reasoning used in the proof of Lemma IV (I, p. 105) shows that $\sigma_{1}$ and $\sigma_{2}$ have as common center the point $z_{4}$ defined by

$$
\left(P_{1}, A_{2}, A_{3}, z_{4}\right)=\lambda \text {. }
$$

The external center of similitude of $S_{2}$ and $S_{3}$ (and which may be the point at infinity) is the external center of similitude or the internal center of similitude for both the pairs of circles $\sigma_{1}, S_{2}$ and $\sigma_{1}, S_{3}$; the internal center of similitude of $S_{2}$ and $S_{3}$ is the external center of similitude for one of the pairs of circles $\sigma_{2}$, $S_{2}$ and $\sigma_{2}, S_{3}$ and the internal center of similitude for the other pair. Neither of the circles $\sigma_{1}$ and $\sigma_{2}$ can coincide with either of the circles $S_{2}$ and $S_{3}$, for the common center of $\sigma_{1}$ and $\sigma_{2}$ is distinct from $A_{2}$ and $A_{3}$. It follows from this fact and the fact that the internal center of similitude of $S_{2}$ and $S_{3}$ differs from their external center of similitude that $\sigma_{1}$ and $\sigma_{2}$ are distinct.

The locus of the points of the variable circle $S_{4}^{\prime}$ is now apparent. Suppose for conveniencè in phraseology that $\sigma_{2}$ is interior to $\sigma_{1}$. The circle $S_{4}^{\prime}$ moves continuously so as to touch both $\sigma_{1}$ and $\sigma_{2}$ and touches every point of both circles. Either $S_{4}^{\prime}$ includes $\sigma_{2}$ or $S_{4}^{\prime}$ does not include $\sigma_{2}$, but in either case the locus of the points of $S_{4}^{\prime}$ is the annular region between and bounded by $\sigma_{1}$ and $\sigma_{2}$. In particular $\sigma_{2}$ (or $\sigma_{1}$ ) may be a null circle, but the locus of the points of $S_{4}^{\prime}$ is never the entire plane.

We have left aside the case that $S_{2}$ and $S_{3}$ have a common center $M$ when $P_{1}$ is transformed to infinity. This situation will not be discussed in detail, but is entirely analogous to the case already treated. The two circles $\sigma_{1}$ and $\sigma_{2}$ have the common center $M$; if $S_{4}$ is chosen in any position and rotated about $M$, the two circles whose common center is $M$ and which are tangent to $S_{4}^{\prime}$ are seen to play the rôle of $\sigma_{1}$ and $\sigma_{2}$. These two circles are the boundaries of the annular region which is the locus of the points of $S_{4}^{\prime}$.

The case where $S_{2}$ or $S_{3}$, say for definiteness $S_{3}$, is a straight line remains to be considered. The circle $S_{4}^{\prime}$ is in every position a straight line parallel to $S_{3}$. When $P_{2}$ varies over $S_{2}$, the locus of the points of $S_{4}^{\prime}$ is seen to be a strip of the plane bounded by two lines parallel to $S_{3}$, which is an annular region. The theorem is then proved for the case that $S_{1}$ is a null circle. We notice that in 
no case can $\sigma_{1}$ and $\sigma_{2}$ simultaneously be null circles, that they never coincide, and that they are tangent when and only when $P_{1}$ lies on $S_{2}$ or $S_{3}$.

6. Theorem VI, the locus resulting from three circles; general case. Our theorem, thus completely proved when $S_{1}$ is a null circle $P_{1}$, will be proved in the general case by determining the locus of the points of the annular region $S_{4}^{\prime \prime}$ bounded by $\sigma_{1}$ and $\sigma_{2}$ as the point $P_{1}$ traces the circle $S_{1}$. We shall suppose for the present that the three circles $S_{1}, S_{2}, S_{3}$ are not coaxial and that no two of them are tangent. It will follow that in Lemma IV (I, p. 105) we can say that as $z_{1}$ is made to vary continuously and in one sense on its locus, the points $z_{1}, z_{2}, z_{3}, z_{4}$ all vary continuously and in one sense on their loci. It should be remarked in connection with Lemma IV (I, p. 105) that it is essential to suppose the variable circle $C$ and the points $z_{1}, z_{2}, z_{3}$ to vary continuously or that at least a suitable convention be made as to the choice of the points $z_{1}, z_{2}, z_{3}$ on $C$ when $C$ is orthogonal to $C_{1}, C_{2}, C_{3}$. For in that position of $C$, the condition that when $C$ is transformed into a straight line the lines tangent to the three circles at $z_{1}, z_{2}, z_{3}$ are all parallel does not determine sufficiently these three points so that $z_{4}$ shall lie on $C_{4}$; and there are in general some extraneous points which enter into our locus if some convention concerning them is not made.

When $P_{1}$ varies and traces $S_{1}, \sigma_{1}$ varies also so as to remain constantly tangent to two fixed circles $\tau_{1}$ and $\tau_{2}$. For consider the circle $C^{\prime}$ through $P_{1}$ and which cuts $S_{1}, S_{2}, S_{3}$ all at the same angle. Choose on $C^{\prime}$ the points $z_{2}$ on $S_{2}$ and $z_{3}$ on $S_{3}$ such that when $C^{\prime}$ is transformed into a straight line the lines tangent to $S_{1}, S_{2}, S_{3}$ at $P_{1}, z_{2}, z_{3}$ are parallel. Then the point $z_{4}$ lies on $\sigma_{1}$ and also lies on the circle $\tau_{1}$ of Lemma IV (I, p. 105) corresponding to the variable circle which cuts the three original circles at equal angles. Moreover when $C^{\prime}$ is transformed into a straight line the lines tangent to $\cdot S_{1}$ at $P_{1}, S_{2}$ at $z_{2}, S_{3}$ at $z_{3}, \sigma_{1}$ at $z_{4}, \tau_{1}$ at $z_{4}$ are all parallel. Hence $\sigma_{1}$ is tangent to $\tau_{1}$ at $z_{4}$.

In a precisely similar way it also appears that $\sigma_{1}$ is tangent to the circle $\tau_{2}$ generated as described in Lemma IV (I, p. 105) corresponding to a variable circle $C^{\prime \prime}$ which cuts $S_{1}$ at an angle supplementary to the angles cut on $S_{2}$ and $S_{3}$. The variable circle $\sigma_{2}$ remains always tangent to two fixed circles $\omega_{1}$ and $\omega_{2}$ corresponding to a variable circle $C^{\prime \prime \prime}$ which cuts $S_{2}$ at an angle supplementary to that cut on $S_{1}$ and $S_{3}$, and a variable circle $C^{\prime \prime \prime \prime}$ which cuts $S_{3}$ at an angle supplementary to that cut on $S_{1}$ and $S_{2}$.

If the variable circle $\sigma_{1}$, is never a null circle, $\tau_{1}$ and $\tau_{2}$ do not intersect and are not tangent; for definiteness suppose $\tau_{2}$ interior to $\tau_{1}$. Whether $\sigma_{1}$ moves so as to include $\tau_{2}$ or so as to exclude $\tau_{2}, \sigma_{1}$ passes through every point of the plane between $\tau_{1}$ and $\tau_{2}$, so every such point is a point of $S_{4}$. A precisely similar remark obtains with reference to ${ }^{\prime} \sigma_{2}$.

If $\sigma_{2}$ is ever a null circle, we shall prove that no arc of either of the circles 
$\omega_{1}$ and $\omega_{2}$ can be a part of the boundary of $S_{4}$. The circles $\omega_{1}$ and $\omega_{2}$ must either intersect or be tangent. Suppose $\sigma_{2}$ never to pass through the point at infinity. When $\sigma_{2}$ is a null circle, $\sigma_{1}$ entirely surrounds $\sigma_{2}$. As $\sigma_{2}$ varies, immediately exterior to it there are always points of the final locus $S_{4}$, for $\sigma_{1}$ and $\sigma_{2}$ can never coincide and they vary continuously. It may occur that $\sigma_{1}$ and $\sigma_{2}$ become tangent, but this can happen only when $P_{1}$ is on $S_{2}$ or $S_{3}$ and thus only at the most at four isolated points. Except possibly at these four points, $\omega_{1}$ and $\omega_{2}$ are entirely embedded in points of $S_{4}$. A similar remark is evidently true for $\sigma_{1}$ and the circles $\tau_{1}$ and $\tau_{2}$, if $\sigma_{1}$ is ever a null circle.

The boundary of $S_{4}$ consists entirely of points of the circles $\tau_{1}, \tau_{2}, \omega_{1}, \omega_{2}$, by Theorem IV. The region $S_{4}$ is closed and its boundary cannot consist in whole or in part of isolated points. We may therefore suppose that at least one of the circles $\sigma_{1}$ or $\sigma_{2}$, say for definiteness $\sigma_{1}$, is never a null circle. If $\sigma_{1}$ passes through every point of the plane, $S_{4}$ is surely the whole plane. If $\sigma_{1}$ does not pass through every point of the plane, we suppose that it does not pass through the point at infinity. If $\sigma_{2}$ is ever exterior to $\sigma_{1}$, it always remains exterior; if ever interior, it always remains interior; for definiteness suppose that $\sigma_{2}$ always remains interior to $\sigma_{1}$. If $\sigma_{1}$ surrounds neither $\tau_{1}$ nor $\tau_{2}, \sigma_{1}$ passes through every point between $\tau_{1}$ and $\tau_{2}$, and every point of $S_{1}^{\prime \prime}$ is a point between those two circles, so $S_{4}$ is the annular region bounded by the non-intersecting circles $\tau_{1}$ and $\tau_{2}$.

If $\tau_{2}$ is interior to $\tau_{1}$ as well as to $\sigma_{1}\left(\sigma_{2}\right.$ constantly interior to $\left.\sigma_{1}\right), \tau_{1}$ is one boundary of $S_{4}$. But no arc of $\tau_{2}$ can be a part of the boundary of $S_{4}$, since except at most for four isolated positions there are points of $S_{4}^{\prime \prime}$ interior and adjacent to $\sigma_{1}$ and hence there are points of $S_{4}$ interior and adjacent to $\tau_{2}$. That is, not more than one of the circles. $\tau_{1}$ and $\tau_{2}$ can be a part of the boundary of $S_{4}$; no arc of either circle is a part of that boundary unless that entire circle is a part of the boundary. The corresponding statement is true for $\omega_{1}$ and $\omega_{2}$.

In any case, then, the boundary of $S_{4}$ is composed of at most two of the circles $\tau_{1}, \tau_{2}, \omega_{1}, \omega_{2} ;$ it follows quite easily from our previous reasoning that these two circles do not intersect, so $S_{4}$ is an annular region.

The foregoing reasoning in proof of Theorem VI is not essentially altered and need not be further considered in detail if any of the circles $\tau_{1}, \tau_{2}, \omega_{1}, \omega_{2}$ is a null circle or if either of the circles $\sigma_{1}$ or $\sigma_{2}$ remains fixed during the motion of $P_{1}$. But on the other hand, it is necessary to note that we have essentially two distinct pairs of circles $\tau_{1}$ and $\tau_{2}$, and $\omega_{1}$ and $\omega_{2}$, or at least that when we speak of the circle $\sigma_{1}$ as tangent to $\tau_{1}$ at $z_{4}^{\prime}$ and tangent to $\tau_{2}$ at $z_{4}^{\prime \prime}$ we are not dealing with two points $z_{4}^{\prime}$ and $z_{4}^{\prime \prime}$ which always coincide and at the same time with two circles $\tau_{1}$ and $\tau_{2}$ which always coincide or are tangent at $z_{4}^{\prime}\left(=z_{4}^{\prime \prime}\right)$. We prove this by choosing $P_{1}$ of such a nature that $C^{\prime}$ does not cut $S_{1}$ orthogonally and is not tangent to $S_{1}$, and such that $\sigma_{1}$ is not a null circle; such choice is possible under 
our restrictions on $S_{1}, S_{2}, S_{3}$. Then $C^{\prime}$ and $C^{\prime \prime}$ cannot cut $S_{1}$ at the same angle and hence are distinct. The lines tangent to $\tau_{1}$ (also to $\sigma_{1}$ ) at $z_{4}^{\prime}$ and to $\tau_{2}$ (also to $\sigma_{1}$ ) at $z_{4}^{\prime \prime}$ cannot coincide, by the property of those tangent lines when $C^{\prime}$ and $C^{\prime \prime}$ are transformed into straight lines. Moreover $\sigma_{1}$ was chosen a non-null circle, so we are led to the conclusion that $z_{4}^{\prime}$ and $z_{4}^{\prime \prime}$ are distinct. This remark is similarly applicable to the points on the circles $\omega_{1}$ and $\omega_{2}$, so Theorem VI is completely proved under our assumption that $S_{1}, S_{2}, S_{3}$ are not coaxial and that no two of them are tangent.

For the more general case which makes no such assumption concerning $S_{1}, S_{2}$, $S_{3}$, ${ }^{*}$ we consider variable auxiliary circles which approach the three given circles, and are such that at no stage are these three variable circles coaxial nor are any two of them tangent. The locus corresponding to the variable circles is always an annular region; it approaches uniformly the locus corresponding to the three given circles; this latter locus is therefore an annular region.

7. Theorem VII, circular boundaries lead to circular boundaries; case of a single null circle. We shall now make use of Theorem VI and its method of proof in the demonstration of our general theorem concerning loci whose bound. aries are entire circles.

THEOREM VII. If the loci of the points $z_{1}, z_{2}, z_{3}$ are, respectively, $T_{1}, T_{2}, T_{3}$, regions each bounded by a finite number of non-intersecting circles, the locus of $z_{4}$ defined by the real constant cross ratio

$$
\lambda=\left(z_{1}, z_{2}, z_{3}, z_{4}\right)
$$

is a region $T_{4}$ also bounded by a finite number of non-intersecting circles. The number of circles bounding $T_{4}$ is not greater than the greatest number of circles bounding any of the regions $T_{1}, T_{2}, T_{3}$. In particular if $T_{1}, T_{2}, T_{3}$ are annular regions, $T_{4}$ is also an annular region; if $T_{1}, T_{2}, T_{3}$ are circular regions, $T_{4}$ is also a circular region.

In counting the number of boundaries of a region, any-region $T_{i}$ which consists merely of the points of a single circle is to be considered as having two boundaries. This is a natural convention, for we may think of $T_{i}$ as the limit of a proper annular region as the two bounding circles approach each other. Some such convention is desirable so that the present theorem shall accord with Theorem VI.

It is of course true that $T_{4}$ may have as many bounding circles as the greatest number of circles bounding any of the regions $T_{1}, T_{2}, T_{3}$. This always occurs

- Theorem VI is extremely easy to prove for three coaxial circles. For three circles through two fixed points or all tangent at a single point the locus is the entire plane. For the other case the circles can be transformed so as to be made concentric. From symmetry about the common center and from the connectedness of the locus (as in I, p. 103) it follows that $S_{4}$ is an annular region. 
$(\lambda \neq 0,1$,or $\infty)$ if for example $T_{1}$ and $T_{2}$ are points; $T_{3}$ is the region or at least one of the regions with the greatest number of bounding circles. If $T_{1}$ and $\dot{T}_{2}$ are sufficiently small, the number of their bounding circles has no effect; $T_{3}$ and $T_{4}$ have the same number of bounding circles.

The cases $\lambda=0,1$, or $\infty$ or that one of the given regions is the entire plane are to be treated as in I, p. 103, and will not be further considered. We shall further suppose for the present, that no two bounding circles of any one of the given regions are tangent, that no three circles, boundaries respectively of the three given regions, are coaxial, and that none of the three given regions is a point or a circle.

The boundary of the locus $T_{4}$ is composed of circles $S_{4}$ or arcs of these circles which are generated by the point $z_{4}$ determined by its cross ratio with the points $z_{1}$ on a circle $S_{1}$ which is a boundary of $T_{1}, z_{2}$ on a circle $S_{2}$ which is a boundary of $T_{2}$, and $z_{3}$ on a circle $S_{3}$ which is a boundary of $T_{3}$, while the circle $S$ through these four points cuts $S_{1}, S_{2}, S_{3}$ all at the same angle or cuts one at angle supplementary to the angle cut on the other two. We shall prove that if an arc of any one of these circles $S_{4}$ is part of the boundary of $T_{4}$, the entire circle $S_{4} i$; part of the boundary of $T_{4}$. For definiteness suppose $S$ to cut $S_{1}, S_{2}, S_{3}$ all at the same angle so that the circle $S_{4}$ with which we are concerned is $\tau_{1}$.

Since no two of the circles bounding $T_{1}$ intersect, there are points of $T_{1}$ adjacent to and all along one side of $S_{1}$, and similarly for the circles $S_{2}$ and $S_{3}$. We shall prove that even if we consider the entire circular region $T_{1}^{\prime}$ lying on that particular side of and bounded by $S_{1}$ as the locus of $z_{1}$ and the regions $T_{2}^{\prime}$ and $T_{3}^{\prime}$ similarly formed from $S_{2}$ and $S_{3}$ as the loci of $z_{2}$ and $z_{3}$, the locus of $z_{4}$ is that circular region $T_{4}^{\prime}$ bounded by $\tau_{1}$ which contains $\tau_{2}, \omega_{1}, \omega_{2}$. Since $T_{1}^{\prime}$ entirely contains $T_{1}, T_{2}^{\prime}$ entirely contains $T_{2}$, and $T_{3}^{\prime}$ entirely contains $T_{3}$, and since every point of $\tau_{1}$ is a point of the locus of $z_{4}$ corresponding to the regions $T_{1}, T_{2}, T_{3}$, it follows that the entire circle $\tau_{1}$ is a boundary of $T_{4}$; there are points $z_{4}$ corresponding to $T_{1}, T_{2}, T_{3}$ lying on one side of and all along $\tau_{1}$, but there are no such points $z_{4}$ lying on the opposite side of $\tau_{1}$.

The circle $\tau_{1}$ is a part of the boundary of the annular region corresponding to the three circles $S_{1}, S_{2}, S_{3}$ as in Theorem VI; otherwise it is surely not part of the boundary for the regions $T_{1}, T_{2}, T_{3}$. Similarly, if we fix a point $P_{1}$ on $S_{1}$ we know that $\sigma_{1}$ must be a part of the boundary of the locus for $P_{1}, S_{2}, S_{3}$.

If we fix $P_{1}$ on $S_{1}$ and $P_{2}$ on $S_{2}$, we have points $z_{3}$ of $T_{3}$ on one side of and all along $S_{3}$, and hence we have points $z_{4}$ of $T_{4}$ on one side of and all along the circle $S_{4}^{\prime}$ of the proof of Theorem VI. Transform $P_{1}$ to infinity. When $P_{2}$ traces $S_{2}$, if $S_{4}^{\prime}$ always lies between $\sigma_{1}$ and $\sigma_{2}$ but surrounds neither, it passes through every point of the annular region between the circles. Moreover, if the points $z_{4}$ of $T_{4}$ just mentioned lie outside of $S_{4}^{\prime}$, no point cf either $\sigma_{1}$ or $\sigma_{2}$ can be a point of the 
boundary of $T_{4}$, and hence no arc of $\tau_{1}$ can be a part of the boundary of $T_{4}$. Then the points $z_{4}$ which correspond to $T_{4}^{\prime}$ must all lie interior to $S_{4}^{\prime}$ and as $P_{2}$ varies on $S_{2}$ we have no new points added to the locus of $z_{4}$ because $T_{4}$ was replaced by $T_{4}^{\prime}$.

When $P_{2}$ moves from $S_{2}$ into $T_{2}$, for example along the line $L$ through $P_{2}$ cutting $S_{2}$ and $S_{3}$ at the same angle, $S_{1}^{\prime}$ moves so as to continue to cut $L$ at the same angle, but is no longer tangent to $\sigma_{1}$ or $\sigma_{2}$. In fact, the intersections of $S_{4}^{\prime}$ with $L$ are the points $z_{4}$ determined by the cross ratio of points $P_{1}$ (fixed), $P_{2}$ (variable on $L$ ), and the fixed intersections of $L$ with $S_{3}$. Motion of $P_{2}$ causes these two intersections to vary. When $P_{2}$ is at either intersection of $I$, with $S_{2}, S_{4}^{\prime}$ is tangent to $\sigma_{1}$ and $\sigma_{2}$; when $P_{2}$ moves on $L$ from one of these intersections to the other in $T_{2}^{\prime}, S_{4}^{\prime}$ varies, but always varies so as to cut $L$ at the same angle, which is the angle cut on $L$ by $\sigma_{1}$. If we assume for definiteness that $S_{4}^{\prime}$ is interior to $\sigma_{1}$, we see that this motion of $S_{4}^{\prime}$ must always keep $S_{4}^{\prime}$ interior to $\sigma_{1}$, and $\sigma_{2}$ is not a part of the boundary of $T_{4}$ nor of $T_{4}^{\prime}$, and that the entire interior of $\sigma_{1}$ belongs to $T_{4}^{\prime}$.

Precisely similar reasoning obtains if $S_{1}^{\prime}$ lies between $\sigma_{1}$ and $\sigma_{2}$ but encloses one circle and not the other, but we shall not give the details. We further omit the detailed treatment if $S_{2}$ or $S_{3}$ is a straight line ( $P_{1}$ being at infinity). In every case we add no new boundary nor take away a part of the boundary of the locus of $z_{4}$ by replacing $T_{2}$ and $T_{3}$ by $T_{2}^{\prime}$ and $T_{3}^{\prime}$, so far as concerns the circle $\tau_{1}$. Moreover the locus of $z_{4}$ in the latter case is not the whole plane unless the locus in the former case is the whole plane.

8. Theorem VII, circular boundaries lead to circular boundaries; general case. We shall now extend this reasoning by considering the locus of the points of the circle $\sigma_{1}$ as $P_{1}$ traces $S_{1}$. Suppose for definiteness that $\tau_{1}$ is exterior to $\tau_{2}$ and that $\sigma_{1}$ lies between the two circles but $\tau_{2}$ is exterior to $\sigma_{1}$. We have assumed a part of $\tau_{1}$ a part of the boundary of $T_{4}$, so the points of $T_{4}$ and hence of $T_{4}^{\prime}$ previously determined lie interior to $\sigma_{1}$. Similarly, a small motion of $P_{1}$ on $S$ and into the interior of $T_{1}$ either moves $\sigma_{1}$ always interior to $\tau_{1}$, or always exterior to $\tau_{1}$; this follows from the continuity of the motion of $\sigma_{1}$ due to the continuous motion of $P_{1}$, and from the properties of the cross ratio determining the intersection of $\sigma_{1}$ with $S$ which are shortly to be considered in detail. Since we are assuming at least an arc of $\tau_{1}$ to be part of the boundary of $T_{4}$, it follows that such small motion of $P_{1}$ always moves $\sigma_{1}$ interior to $\tau_{1}$.

Continuous motion of $P_{1}$ in one sense along $S$ and in $T_{1}^{\prime}$ from one intersection of $S$ with $S_{1}$ to the other intersection causes $\sigma_{1}$ to move. Each intersection of $S$ with $\sigma_{1}$ is a point $z_{4}$ determined by its cross ratio with $P_{1}$, an intersection of $S$ with $S_{2}$, and an intersection of $S$ with $S_{3}$. The angle of intersection of $S$ and $\sigma_{1}$ does not change, and this is the same as the angle of intersection of $S$ and $\tau_{1}$. 
In its initial and final positions $\sigma_{1}$ is interior to $\tau_{1}$; it therefore follows (in fact becomes evident if $S$ is transformed into a straight line) that every point of $\sigma_{1}$ remains always interior to $\tau_{1}$.

Determination of the locus of $z_{4}$ in this manner gives us the entire region $T_{4}^{\prime}$; we have shown every point of $T_{4}^{\prime}$ to lie interior to or on $\tau_{1}$. Every point interior to or on $\tau_{1}$ is on a circle $S$ and is interior to or on the circle $\sigma_{1}$ for some choice of $P_{1}$ in $T_{1}^{\prime}$ and hence $T_{4}^{\prime}$ is the interior (boundary included) of $\tau_{1}$.

There is a possibility which could conceivably arise in this proof, namely that for some positions of $S$ that circle does not cut $S_{1}$ and that for some point $P_{1}$ interior to $T_{1}^{\prime}$ but not on a circle $S$ cutting $S_{1}^{*}$, the circle $\sigma_{1}$ should be completely or partially exterior to $\tau_{1}$; our reasoning as given does not permit this possibility. If there is one of the regions $T_{1}^{\prime}, T_{2}^{\prime}, T_{3}^{\prime}$ for which the circle $S$ passes through every point, we change our notation if necessary so that that region shall be $T_{1}^{\prime}$, and our proof is valid as given. If there is no region $T_{1}^{\prime}, T_{2}^{\prime}, T_{3}^{\prime}$ through every point of which passes a circle $S$ cutting $S_{1}, S_{2}, S_{3}$, it follows from the proof of Lemma IV (I, p. 105) that a point $P_{1}$ on $S_{1}$ but on neither $S_{2}$ nor $S_{3}$ can be chosen which lies in both $T_{2}^{\prime}$ and $T_{3}^{\prime}$. It follows from the development of $\S 7$ that no point of either $\sigma_{1}$ or $\sigma_{2}$ can be a boundary point of $T_{4}^{\prime}$, so the possibility suggested need not be considered further.

The case wnere $\sigma_{1}$ lies between $\tau_{1}$ and $\tau_{2}$ but separates those two circles needs to be considered in detail, but only slight modifications in the reasoning are necessary and these are left to the reader. This completes the proof that $\tau_{1}$ is the boundary of $T_{4}^{\prime}$ and hence part of the boundary of $T_{4}$. There are points $z_{4}$ of $T_{4}$ all along one side of and adjacent to $\tau_{1}$, so no other bounding circle of $T_{4}$ (the entire circle necessarily part of the boundary of $T_{4}$ ) can intersect $\tau_{1}$.

We have made the assumption that no two bounding circles of any one of the regions $T_{i}$ are tangent, that no three circles, boundaries respectively of the three

* A similar possibility should have been pointed out in I, pp. 111-112 in proving that (notation of I) either the entire exterior or the entire interior of each of the circles $C_{4}^{\prime}, C_{4}^{\prime \prime}, C_{4}^{\prime \prime \prime}, C_{4}^{\prime \prime \prime}$ belongs to the region $C_{4}$. For definiteness suppose this possibility to arise in connection with Lemma IV (I, p. 105), Case I; we consider the configuration simplified by transformation as in $\mathrm{I}, \mathrm{p}$. 106. 'The statement desired is evident if the regions $C_{1}, C_{2}, C_{3}$ are all interior to their bounding circles, so we may suppose one of these regions, say $C_{3}$, exterior to its bounding circle $C_{3}$. We wish to prove a point $z_{4}$ exterior to $C_{6}^{\prime}$ a point of the final envelope $C_{4}$ and need consider only points $z_{4}$ on circles $C$ which do not cut $C_{1}, C_{2}, C_{3}$. Then there is a circle through 24 which cuts $C_{1}$ and $C_{2}$ and which lies entirely in the region $C_{3}$. We may choose $z_{1}$ and $z_{2}$ on this circle and lastly $z_{3}$, so that these three points are in their proper envelopes and have the proper cross ratio with $z_{4}$.

The entire reasoning given in $I, \mathrm{pp} .110-112$, to prove that arcs of but one of the circles $C_{1}^{(i)}$ can be a part of the boundary of $C_{4}$ is no longer strictly necessary, for the proof of Theorem VII of the present paper contains a proof of Theorem I. Moreover, the reasoning of I, pp. $110-112$, can be replaced by the more simple and elegant argument used in $\$ 6$ (or even \$15) of a paper by the writer shortly to appear in these $\mathrm{Tr}$ a n a c t i o ns. 
given regions, are coaxial, and that none of the given regions is a point or a circle. To extend our result to include these special cases, we consider a sequence of sets of three regions which satisfy our restrictions but which approach the more special given regions; such an auxiliary sequence can always be constructed. Then the limit of the locus of $z_{4}$ for the variable regions is the locus of $z_{4}$ for the limit regions, from which it follows that for the limit case the locus $T_{4}$ is bounded by a finite number of entire circles.

9. Theorem VII, number of circular boundaries. It remains for us to prove that the number of circles bounding $T_{4}$ is not greater than the greatest number of circles bounding any of the regiors $T_{1}, T_{2}, T_{3}$. In the detailed proof we restrict the boundaries of the original regions as before; this restriction is raised by the limiting process previously used and need not be mentioned further.

We keep $P_{1}$ ( $z_{1}$ in any position) fixed for the moment, and suppose that the point at infinity is not a point of the locus $T_{4}^{\prime \prime}$ corresponding to the points $z_{2}$ of $T_{2}$ and $z_{3}$ of $T_{3}$. Consider the mechanism used in the proof of Theorem VI; first suppose $S_{4}^{\prime}$ to lie between $\sigma_{1}$ and $\sigma_{2}$ and $\sigma_{2}$ to be interior to $\sigma_{1}$ but not interior to $S_{4}^{\prime}$. Under the assumption that $\sigma_{1}$ is part of the boundary of $T_{4}^{\prime \prime}$, when $P_{2}$ traces $S_{2}, S_{4}^{\prime}$ moves tangent to $\sigma_{1}$ and $\sigma_{2}$. If $\Sigma_{4}^{\prime}$ is a circle which is obtained from the proper cross ratio from $P_{1}, P_{2}$, and the points of a bounding circle $\Sigma_{3}$ of $T_{3}$ other than $S_{3}$, then $\Sigma_{4}^{\prime}$ is interior to $S_{4}^{\prime}$. During the motion of $P_{2}$ on $S_{2}$, $\Sigma_{4}^{\prime}$ cannot trace a boundary of $T_{4}^{\prime \prime}$. Moreover, if $P_{2}$ is moved continuously, $\Sigma_{4}^{\prime}$ moves continuously; $\Sigma_{4}^{\prime}$ can never be made to surround a lacuna in $T_{4}^{\prime \prime}$, from which it follows that $\Sigma_{3}$ in combination with any bounding circle of $T_{2}$ cannot lead to a boundary of $T_{4}^{\prime \prime}$. Bounding circles of $T_{4}^{\prime \prime}$ can proceed only from combination of $S_{3}$ with bounding circles of $T_{2}$, so the number of bounding circles of $T_{4}^{\prime \prime}$ is not greater than the number of bounding circles of $T_{2}$.

Second, suppose $S_{4}^{\prime}$ to lie between $\sigma_{1}$ and $\sigma_{2}$ and $\sigma_{2}$ to be interior to both $\sigma_{1}$ and $S_{4}^{\prime}$. When $\sigma_{1}$ is supposed as before part of the boundary of $T_{4}^{\prime \prime}$, the circle $\Sigma_{4}^{\prime}$ must as before be interior to $S_{4}^{\prime}$. There is a circle $\Sigma_{4}^{\prime}$ interior to $S_{4}^{\prime}$ corresponding to each of the $m$ bounding circles $\Sigma_{3}$ of $T_{3}$ (other than $S_{3}$ ), and in the region traced out by these circles as $P_{2}$ moves around $S_{2}$ there are at most $m$ lacunae, each constantly surrounded by a circle $\Sigma_{4}^{\prime}$. When $P_{2}$ is made to trace the whole of $T_{2}$, one or more of these lacunae may disappear, but none of them can divide to make two or more lacunae in $T_{4}^{\prime \prime}$. It follows that the number of bounding circles of $T_{4}^{\prime \prime}$ is not greater than the number of bounding circles of $T_{3}$.

The case that $\sigma_{2}$ is a point leads simply to one bounding circle of $T_{4}^{\prime \prime}$, so in every case $T_{4}^{\prime \prime}$ is bounded by a number of circles not greater than the greater number of bounding circles of $T_{2}$ and $T_{3}$.

If the reasoning just used is again applied regarding the locus of $T_{4}^{\prime \prime}$ as $P_{1}$ varies over $T_{1}$, it is seen that $T_{4}$ is bounded by a number of circles not greater 
than the greater number of bounding circles of $T_{1}$ and $T_{4}^{\prime \prime}$, which is not greater than the greatest number of bounding circles of $T_{1}, T_{2}, T_{3}$, so the proof of Theorem VII is complete. It is also true that if $T_{1}, T_{2}, T_{3}$ are each bounded by at most a countable infinity of non-intersecting circles then $T_{4}$ is also bounded by at most a countable infinity of non-intersecting circles.

10. Theorem VII, ruler-and-compass construction for boundaries. As in Theorem I, the circles which bound the region $T_{4}$ of Theorem VII can be constructed by ruler and compass whenever $\lambda$ is rational or is given geometrically; indeed this follows from the fact that any circle $C_{4}$ of Lemma IV (I, p. 105) can be so constructed; see I, p. 105, footnote. We also have a test for determining whether or not a given circle $S_{4}$ (in the notation of $\$ 7$ ) is actually a part of the boundary of $T_{4}$. If none of the regions $T_{1}, T_{2}, T_{3}$ reduces to the points of a circle, we need merely suppose the circles $S_{1}, S_{2}, S_{8}$ to bound entire circular regions which lie on the same side of those circles as the given regions; then $S_{4}$ is a boundary of $T_{4}$ when and only when the locus of $z_{4}$ in the simplified situation is a circular region bounded by $S_{4}$. A test for this latter fact has already been determined in I, p. 112. If any of the regions $T_{1}, T_{2}, T_{2}$ reduces to the points of a circle, we may determine in what sense to consider the corresponding circular regions to lie by an investigation such as that of $\$ 7 .^{*}$

To be sure, the test for determining whether $S_{4}$ can be a boundary of $T_{4}$ was proved only under certain restrictions on the bounding circles of $T_{1}, T_{2}, T_{3}$, but the test can be applied whether those restrictions are satisfied or not. Thus, if the circular regions which lie on the same side of $S_{1}, S_{2}, S_{3}$ as do the given regions $T_{1}, T_{2}, T_{3}$ lead to a region $T_{4}^{\prime}$ bounded by a circle $\tau_{1}$ and which is not the whole plane, the original regions lead to a region $T_{4}$ bounded by the entire circle $\tau_{1}$ and which is not the whole plane. On the other hand, if a circle $S_{4}$ is actually a boundary of $T_{4}$, there must be a circle which bounds the locus of $z_{4}$ of the approximating sequence and which approaches $S_{4}$. There are therefore entire variable circular regions $T_{1}^{\prime}, T_{2}^{\prime}, T_{3}^{\prime}$ corresponding which lead to a locus of $z_{4}$ not the whole plane; these approach entire circular regions formed in the manner described from certain boundaries of $T_{1}, T_{2}, T_{3}$, and which lead to a locus of $z_{4}$ not the whole plane. It follows that the test to determine whether $S_{4}$ is a boundary of $T_{4}$ is valid in every case. If any of the given regions is a circle, the results in the given case may also be indicated by approximating non-special cases.

* One particular case of three circles $S_{1}, S_{2}, S_{3}$ can be treated directly, namely, where one of those circles separates the intersections of the other two; compare $I, p .111$. The locus of $s_{4}$ is always the entire plane. For any given point $P$ of the plane there can be determined points $z_{1}, z_{2}, z_{2}$ on $S_{1}, S_{2}, S_{2}$ such that their cross ratio with $P$ has a value very small and positive; these points fan be moved continuously on their respective circles so that this cross ratio increases in value and becomes less than but nearly equal to unity. Then these points can be chosen so that $\lambda$ takes any value between zero and unity; similarly, so that it takes any real value. 
Great care should be used in determining loci and limits of loci of this nature if boundaries of the three given regions are tangent at a single point, for it may be merely by virtue of the fact that when $z_{1}, z_{2}, z_{3}$ coincide $z_{4}$ is undetermined that the locus of $z_{4}$ is the entire plane. Thus, if two finite points $z_{1}$ and $z_{2}$ have as their common locus a half-plane, the locus of the mid point $z_{4}$ of their segment is also that half-plane. When we consider not the problem of the ordinary ratio but that of the cross ratio of the two giren points and the mid point of their segment with the point at infinity we are compelled to admit the point at infinity as a possibility for $z_{1}$ and $z_{2}$ and that the entire plane is the locus of $z_{4}$ (compare $I ; \mathbf{p}$. 111). For there can be found two variable points $z_{1}$ and $z_{2}$ such that $z_{4}$ is the mid point of their segment and which approach the point at infinity. When these two points coincide at infinity we ought therefore to consider that the four limit points have the proper cross ratio. But for an arbitrary point $z_{4}$ of the plane there cannot be found two variable finite points $z_{1}$ and $z_{2}$ in their proper loci, such that $z_{4}$ is the mid point of their segment and which approach the point at infinity. This phenomenon can occur only if the three regions haxe but a single common point and if the respective boundaries are tangent at that point.

This difference of behavior of ratios and cross ratios corresponds indeed to a difference of behavior in our original problem of the roots of the jacobian, as compared with the problem of the roots of the derivative of a polynomial. For most purposes these problems are equivalent if one of the ground forms has all its roots at infinity and the other has as its roots the roots of the polynomial considered. If all the roots of both ground forms coincide, the jacobian vanishes identically. Hence if in Theorem III (I, p. 112) the loci of $m$ roots of $f_{1}$ and the remaining $p_{1}-m$ roots of $f_{1}$ are two coincident half-planes, and if $f_{2}$ has all its roots coincident at infinity, the locus of the roots of the jacobian is the entire plane. But if these two coincident half-planes are the loci of $m$ roots of a polynomial and the remaining $p_{1}-m$ roots of that polynomial, and if the polynomial is not allowed to have infinite roots, the locus of the roots of the derivative of this polynomial is this same half-plane instead of the entire plane.

We have assumed in Theorem VII not only that the boundaries of $T_{1}, T_{2}, T_{3}$ are entire circles but also that no two of the bounding circles of one of these regions cut each other. If this latter part of the hypothesis is omitted, it is not true that $T_{4}$ is bounded by entire circles. Consider for example $T_{1}$ the point at infinity, $T_{2}$ the two finite crescents bounded by two intersecting circles $S_{2}^{\prime}$ and $S_{2}^{\prime \prime}$, and $T_{3}$ the interior and boundary of a circle $S_{3}$ whose center is $A$ and whose radius is small in comparison with the radii of $S_{2}^{\prime}$ and $S_{2}^{\prime \prime}$. Choose $\lambda$ so that when $z_{1}$ is at infinity $z_{4}$ lies midway between the finite points $z_{2}$ and $z_{3}$.

The locus of $z_{4}$ may be determined by fixing $z_{2}$, determining the locus of $z_{4}$ while $z_{3}$ varies over $T_{3}$, and then allowing $z_{2}$ to vary over $T_{2}$. That is, we shrink $T_{2}$ toward $A$ as center of similitude in the ratio $1: 2$, and determine the locus of the 
points of a small circle whose radius is half the radius of $S_{3}$ and whose center varies all over this new configuration. The locus $T_{4}$ is therefore bounded by arcs of four different circles; no entire circle is a part of its boundary.

11. Successive application of Theorem $I$ in determination of loci. An evident way of generalizing Theorem $I$ is by successive application of that theorem. Thus, suppose we have $n$ sets of the circular regions of Theorem I, $C_{k}^{(i)}(k=1,2,3,4 ; i=1,2, \ldots, n)$. Any point $z_{4}$ which corresponds to points $z_{k}(k=1,2,3)$ in all the regions $C_{k}^{(i)}(i=1,2, \ldots, n)$ is located in all the regions $C_{4}^{(i)}(i=1,2, \ldots, n)$, so the region $T_{4}$ which is the locus of points $z_{4}$ determined by points $z_{k}$ in the regions $T_{k}$ common to all the $C_{k}^{(i)}(i=1,2, \ldots, n ; k=1,2,3)$ is contained in all the regions $C_{4}^{(i)}$. But ordinarily $T_{4}$ will not be the entire region common to this last set of regions. A simple example is the case, $\lambda=\frac{1}{2}$ :

$$
\begin{array}{lll}
C_{1}^{(1)}: \infty & C_{1}^{(2)}: \infty & T_{1}: \infty \\
C_{2}^{(1)}:\left|z_{2}+2\right| \leqq 1 & C_{2}^{(2)}:-2 & T_{2}:-2 \\
C_{3}^{(1)}: 2 & C_{3}^{(2)}:\left|z_{3}-2\right| \leqq 1 & T_{3}: 2 \\
C_{4}^{(1)}:\left|z_{4}\right| \leqq \frac{1}{2} & C_{4}^{(2)}:\left|z_{4}\right| \leqq \frac{1}{2} & T_{4}: 0 .
\end{array}
$$

A particularly interesting case such that $T_{4}$ is the entire region common to the $C_{4}^{(i)}$ occurs when all the sets of circles $C_{1}^{(i)}, C_{2}^{(i)}, C_{3}^{(i)}, C_{4}^{(i)}$ come under a single case (e. g., Case I) of Lemma IV. (I, p. 105), where these circles have a single coaxial system of circles $C$ cutting them all at the same angle or a definite set or sets at angles supplementary to the angles cut on the other sets, and where all the circular regions $C_{k}^{(i)}$ for one value of $i$ have the same disposition with respect to their bounding circles as the corresponding regions for every other value of $i$. It follows from the reasoning of $I, \mathrm{pp} .111-112$, as supplemented in a footnote to $\$ 8$ of the present paper, that every point of the region common to all the $C_{4}^{(i)}$ is a point of the locus of $z_{4}$, so that common region is precisely $T_{4}$. The circle $C$ then cuts the boundaries of $T_{1}, T_{2}, T_{3}, T_{4}$ all at the same angle or one or two of those regions at an angle supplementary to the angle cut on the others. Our notation here has supposed the sets of circles $C_{k}^{(i)}$ finite in number, but of course that is unnecessary.

A special case of this last result will be considered in some detail, where a set of regions $C_{k}^{(i)}$ is one and the same point for all values of $i$. We first state explicitly a special case of Theorem I, which corresponds to a theorem given in I, p. 115, and which is proved explicitly in $S$.

THEOREM VIII. If the loci of the points $z_{1}$ and $z_{2}$ are the interiors and boundaries of the circles $C_{1}$ and $C_{2}$ whose centers are $a_{1}$ and $a_{2}$ and radii $\tau_{1}$ and $\tau_{2}$, respectively, then the locus of the point

$$
z=\frac{m_{2} z_{1}+m_{1} z_{2}}{m_{1}+m_{2}}
$$


which divides the segment $\left(z_{1}, z_{2}\right)$ in the real constant ratio $m_{1}: m_{2}\left(m_{1} m_{2}>0\right)$ is the interior and boundary of the circle $C$ whose center is

$$
\frac{m_{2} a_{1}+m_{1} a_{2}}{m_{1}+m_{2}}
$$

and radius

$$
\frac{m_{2} \tau_{1}+m_{1} \tau_{2}}{m_{1}+m_{2}}
$$

The point

$$
\frac{\tau_{2} a_{1}-\tau_{1} a_{2}}{\tau_{2}-\tau_{1}}
$$

(which may be the point at infinity) is an external center of similitude for any pair of the circles, $C_{1} C_{2}, C$.

We next prove a generalization of Theorem VIII:

THEOREM IX. If two finite or infinite convex regions $C_{1}$ and $C_{2}$ having an external center of similitude $P$ (which may be the point at infinity) are the loci of two points $z_{1}$ and $z_{2}$, respectively, then the locus of the point $z$ which divides the segment $\left(z_{1}, z_{2}\right)$ in the real constant ratio $m_{1}: m_{2}\left(m_{1} m_{2}>0\right)$ is a region $C$ such that $P$ is an external center of similitude for any pair of the regions $C_{1}, C_{2} ; C$.

In Theorem IX, we tacitly assume $C_{1}$ and $C_{2}$ to be closed regions bounded by regular curves. We formulate the proof of the theorem for the case that $P$ is finite, but a very slight change in phraseology will give the proof when $P$ is infinite.

Consider a line $L$ through $P$ cutting the boundary of $C_{1}$ in points $A_{1}$ and $A_{1}^{\prime}$ and the boundary of $C_{2}$ in corresponding points $A_{2}$ and $A_{2}^{\prime}$. If $C_{1}$ and $C_{2}$ are infinite, $A_{1}^{\prime}$ and $A_{2}^{\prime}$ are to be considered the point at infinity. When $L$ rotates about $P$, the points $A$ and $A^{\prime}$ which divide the segments $\left(A_{1}, A_{2}\right)$ and $\left(A_{1}^{\prime}\right.$, $\left.A_{2}^{\prime}\right)$, respectively, in the ratio $m_{1}: m_{2}$ trace the boundary of a convex region $C$ -such that $P$ is an external center of similitude for any pair of the regions $C_{1}, C_{2}, C$. We shall prove $C$ to be the locus of $z$. Any point $z$ of $C$ is a point of the locus, for we need merely choose $z_{1}$ and $z_{2}$ on the line $P z$ and having the same relative situation in $C_{1}$ and $C_{2}$ as $z$ in $C$. In order to prove that every point $z$ is in $C$ we shall use a preliminary theorem which is a special case of Theorem IX and a limiting case of Theorem VIII:

THEOREM X. If two half-planes $\pi_{1}$ and $\pi_{2}$ bounded by two parallel lines $\lambda_{1}$ and $\lambda_{2}$ and lying on the same side of those lines are the loci of two points $z_{1}$ and $z_{2}$, respectively, then the locus of the point $z$ which divides the segment $\left(z_{1}, z_{2}\right)$ in the real constant ratio $m_{1}: m_{2}\left(m_{1} m_{2}>0\right)$ is a half-plane $\pi$ bounded by a line $\lambda$ parallel to $\lambda_{1}$ and $\lambda_{2} ; \pi$ lies on the same side of $\lambda$ as $\pi_{1}$ and $\pi_{2}$ of $\lambda_{1}$ and $\lambda_{2}$. Any line which 
cuts $\lambda_{1}, \lambda_{2}, \lambda$ cuts them in points $z_{1}, z_{2}$, $z$ such that $z$ divides the segment $\left(z_{1}, z_{2}\right)$ in the ratio $m_{1}: m_{2}$.

We prove Theorem $\mathrm{X}$ in the same spirit as Theorem VIII was proved in S. We use rectangular coördinates $z_{1}=x_{1}+i y_{1}, z_{2}=x_{2}+i y_{2}, z=x+i y$, and suppose the regions $\pi_{1}$ and $\pi_{2}$ to be $x_{1} \geqq a_{1}$ and $x_{2} \geqq a_{2}$, respectively. The region $\pi$ is

$$
x \geqq \frac{m_{2} a_{1}+m_{1} a_{2}}{m_{1}+m_{2}} \text {. }
$$

For given $z_{1}$ and $z_{2}$ in their proper loci, the formula

$$
x=\frac{m_{2} x_{1}+m_{1} x_{2}}{m_{1}+m_{2}}
$$

shows at once that $z$ is in $\pi$. If $z$ is given in $\pi$ we have only to choose

$$
y_{1}=y_{2}=y, \quad x_{1}=a_{1}-\frac{m_{2} a_{1}+m_{1} a_{2}}{m_{1}+m_{2}}+x, x_{2}=a_{2}-\frac{m_{2} a_{1}+m_{1} a_{2}}{m_{1}+m_{2}}+x,
$$

and we shall have $z_{1}$ and $z_{2}$ in $\pi_{1}$ and $\pi_{2}$ and such that $z$ divides their segment in the ratio $m_{1}: m_{2}$. The axis of reals cuts $\lambda_{1}, \lambda_{2}, \lambda$ in points $z_{1}, z_{2}, z$ such that $z$ divides the segment $\left(z_{1}, z_{2}\right)$ in the ratio $m_{1}: m_{2}$, and hence that is true of any other line which cuts $\lambda_{1}, \lambda_{2}, \lambda$.

Theorem $\mathrm{X}$ is to be used in proving that every point $z$ of Theorem IX lies in $C$. Through the point $A_{1}$ draw a line $\lambda_{1}$ which does not cut $C_{1}$ and through $A_{2}$ a parallel line $\lambda_{2}$, where $A_{1}$ and $A_{2}$ have the same significance as before. There is a half-plane $\pi_{1}$ bounded by $\lambda_{1}$ which contains all of $C_{1}$ and a corresponding half-plane $\pi_{2}$ bounded by $\lambda_{2}$ which contains all of $C_{2}$. The corresponding halfplane $\pi$ bounded by the line $\lambda$ through $A$ contains all of $C$, from which it follows that any point $z$ of Theorem IX lies in $\pi$. This is always true as $L$ rotates about $P$ and as $\lambda_{1}, \lambda_{2}, \lambda$ envelop $C_{1}, C_{2}, C_{3}$, so $z$ lies in $C$ and Theorem IX is completely proved.

Our proof depends essentially on the fact that $C_{1}$ and hence also $C_{2}$ and $C$ are convex, since we have passed a line $\lambda_{1}$ through an arbitrary point $A_{1}$ of the boundary of $C_{1}$ and supposed $C_{1}$ to lie entirely on one side of that line. If $C_{1}$ is not convex not only does the proof break down, but the theorem is false and we can even show that the locus of $z$ cannot be the region $C$ found as previously described. There are two points $U_{1}$ and $V_{1}$ on the boundary of $C_{1}$ such that no point of the segment $U_{1} V_{1}$ belongs to $C_{1}$. The analogous segment $U_{2} V_{2}$ has no point in $C_{2}$. Denote by $U$ and $V$ the points on the boundary of $C$ which divide the segments $U_{1} U_{2}$ and $V_{1} V_{2}$ in the ratio $m_{1}: m_{2}$; the segment $U V$ has no point in $C$. The lines $U_{1} V_{1}, U_{2} V_{2}, U V$ are all parallel and the point $z$ which divides the segment $U_{2} V_{1}$ in the ratio $m_{1}: m_{2}$ lies on the segment $U V$ and hence is exterior to the region $C$. 
We terminate our study of the loci connected with cross ratios by stating without further proof the theorem analogous to Theorem IX if $m_{1} m_{2}<0$ :

THEOREM XI. If two finite or infinite convex regions $C_{1}$ and $C_{2}$ having a finite internal center of similitude $P$ are the loci of two points $z_{1}$ and $z_{2}$, respectively, then the locus of the point $z$ which divides the segment $\left(z_{1}, z_{2}\right)$ in the real constant ratio $m_{1}: m_{2}\left(m_{1} m_{2}<0\right)$ is a region $C$ such that $P$ is an external center of similitude for $C_{1}$ and $C$ (or $C_{2}$ and $C$ ) and an internal center of similitude for $C_{2}$ and $C$ (or $C_{1}$ and $\left.C\right)$.

If $C_{1}$ and $C_{2}$ are not convex but satisfy the other conditions of the theorem, the corresponding region $C$ cannot be the locus of $z$.

12. Application to the location of the roots of the jacobian of two binary forms. Theorem I was originally proved in I not for its own sake but for application to the study of the location of the roots of the jacobian of two binary forms. Thus there can be proved (I, p. 114):

THEOREM XII. Let $f_{1}$ and $f_{2}$ be binary forms of degrees $p_{1}$ and $p_{2}$, respectively, and let the circular regions $C_{1}, C_{2}, C_{3}$ be the respective loci of $m$ roots of $f_{1}$, the remaining' $p_{1}-m$ roots of $f_{1}$, and all the roots of $f_{2}$. Denote by $C_{4}$ the circular region which is the locus of points $z_{4}$ such that

$$
\left(z_{1}, z_{2}, z_{3}, z_{4}\right)=\frac{p_{1}}{m}
$$

when $z_{1}, z_{2}, z_{3}$ have the respective loci $C_{1}, C_{2}, C_{3}$. Then the locus of the roots of the jacobian of $f_{1}$ and $f_{2}$ is composed of the region $C_{4}$ together with the regions $C_{1}, C_{2}, C_{3}$, except that among the latter the corresponding region is to be omitted if any of the numbers $m, p_{1}-m, p_{2}$ is unity. If a region $C_{i}(i=1,2,3,4)$ has no point in common with any other of those regions which is a part of the locus of the roots of the jacobian, it contains precisely $m-1, p_{1}-m-1, p_{2}-1$, or 1 of those roots according as $i=1,2,3$, or 4 .

Theorem XII can be generalized so as to give applications for some of the results of the present paper. Thus we have directly:

THEOREM XIII. Let $f_{1}$ and $f_{2}$ be binary forms of degrees $p_{1}$ and $p_{2}$, respectively, and let regions $T_{1}, T_{2}, T_{8}$ be the loci of $m$ roots of $f_{1}$, the remaining $p_{1}-m$ roots of $f_{1}$, and all the roots of $f_{2}$, these three sets of roots always to be contained in three variable circular regions all points of which are points of $T_{1}, T_{2}, T_{3}$, respectively. Denote by $T_{4}$ the locus of points $z_{4}$ such that

$$
\left(z_{1}, z_{2}, z_{3}, z_{4}\right)=\frac{p_{1}}{m}
$$

when $z_{1}, z_{2}, z_{3}$ have the respective loci $T_{1}, T_{2}, T_{3}$. Then the locus of the roots of the jacobian of $f_{1}$ and $f_{2}$ is composed of the region $T_{4}$ together with the regions $T_{1}, T_{2}, T_{3}$, except that among the latter the corresponding region is to be omitted if any of the 
numbers $m, p_{1}-m, p_{2}$ is unity. If a region $T_{i}(i=1,2,3,4)$ has no point in common with any other of those regions which is a part of the locus of the roots of the jacobian, it contains precisely $m-1, p_{1}-m-1, p_{2}-1$, or 1 of those roots according as $i=1,2,3$, or 4 .

It is in connection with Theorem XIII that most of the results of the present paper are to be considered so far as concerns their application to the location to the roots of the jacobian. It should be noticed, however, that Theorem XIII gives no better indication of the location of the roots of the jacobian of particular fixed forms than does Theorem XII, but may give a better indication than Theorem XII of the location of the roots of the jacobian of two forms whose roots vary in particular ways. The results of $\$ 11$ can also be used independently of Theorem XIII in establishing slight extensions of Theorem XII which do apply to the jacobian of two fixed forms; this application will be made in a later paper.

HaRvard UntVersity,

Cambridge, Mass. 